\title{
$\beta$-D-Glucan Assay in Diagnosis and Monitoring the Systemic Candidiasis in a Rat Model
}

\author{
Hossein Khodadadi ${ }^{1}$; Hossein Mirhendi ${ }^{2, *}$; Koichi Makimura ${ }^{3}$; Kazuo Satoh ${ }^{3}$; Ladan \\ Karimi ${ }^{4}$; Shahrokh Izadi ${ }^{2}$ \\ ${ }_{1}^{1}$ Department of Medical Parasitology and Mycology, School of Medicine, Shiraz University of Medical Sciences, Shiraz, IR Iran \\ ${ }_{2}^{2}$ Department of Medical Parasitology and Mycology, School of Public Health, National Institute of Health Research, Tehran University of Medical Sciences, Tehran, IR Iran \\ ${ }^{3}$ Institute of Medical Mycology, Teikyo University, Tokyo, Japan \\ ${ }^{4}$ Center of Medical Commission and Occupational Medicine, Social Security Organization, Esfahan, IR Iran \\ ${ }^{*}$ Corresponding author: Hossein Mirhendi, Department of Medical Parasitology and Mycology, School of Public Health, Tehran University of Medical Sciences, Tehran, IR Iran. Tel/ \\ Fax:+98-2188951392, E-mail: mirhendi@tums.ac.ir \\ Received: January 13, 2013; Revised: April 24, 2013; Accepted: June 15, 2013
}

\begin{abstract}
Background:Determination of $\beta$-D-Glucan (BDG) in the serum aids to diagnose the invasive fungal infections. The current study evaluated the diagnostic potential value of BDG assay in monitoring the disease in experimental systemic candidiasis in a rat model. The results can provide a useful preliminary data to improve this approach in developing countries.

Objectives: The present study aimed to evaluate $\beta$-D-Glucan assay in diagnosis and monitoring the systemic candidiasis in a rat model. Materials and Methods: Twenty one rats were infected with $10^{6}$ Candida albicans blastospore per rat. Twelve rats were considered as the negative controls (six immunocompromised rats without infection and six intact rats). During a week, every 24 hours the BDG sera level was determined by both Fungitell and Wako kits. To confirm the systemic infection in each rat, the suspensions of their internal organs were cultivated on agar plates and the number of colony forming units(CFU) of C. albicans was counted.

Results: All the infected rats were positive with BDG tests. An increasing level of BDG was observed during early days after injection. The cutoff value for discrimination of BDG positive sera was obtained from the negative sera by the Fungitell kit. The sensitivity, specificity, positive and negative predictive values assessed for the Fungitell kit were $95 \%, 66.6 \%, 90.47 \%$ and $80 \%$, respectively. These criteria for those of Wako were $90 \%, 83.3 \%, 94.7 \%$ and $71.4 \%$, respectively.

Conclusions: While BDG assay seems to be a sensitive and specific adjunctive tool to diagnose and monitor the experimental systemic candidiasis, it seems that measuring the positive cutoff value in different laboratory conditions is necessary for favorable establishment of these tests.

Keywords:Beta-D-glucan; Systemic candidiasis; Diagnosis
\end{abstract}

\section{Background}

Invasive fungal infections (IFIs) have increased in recent decades. Widespread use of broad spectrum antibiotics, immunosuppressive drugs, use of indwelling devices, and increased population of patients undergoing organ transplantation are the major causes of this rise (1,2). Invasive aspergillosis and candidiasis are the most important and common IFIs in immunocompromised patients. The mortality rate of patients infected with these fungi is high and up to 50\% $(3,4)$. Therefore a non-invasive, rapid, and reliable diagnostic tool, as well as an early and targeted treatment to monitor the disease, and avoid unnecessary use of potentially toxic and costly drugs are critical. Conventional culture-based methods such as blood culture variants lack enough sensitivity, therefore in the best conditions they are positive in only less than $50 \%$ of candidiasis, and $10 \%$ of invasive aspergillosis cases, and it takes several days to achieve the results (5-7). Recently non-culture based methods to detect fungal elements in at-risk patients have been described. Enzyme Immunoassays (EIA) to detect fungal antigens, polymerase chain reaction (PCR) for specific DNA, and assays detecting fungal components and metabolites such as mannan, glucan, or enolase have been reported (6, 8-10). However, even by using these approaches, reliable diagnoses of IFIs are still problematic.

(1-3) Beta-D-Glucan (BDG) is a major structural component of many fungal cell walls excluding Zygomycetes and Cryptococcus neoformans which release no or little BDG in the human serum $(11,12)$. (1-3) Beta-D-Glucan and bacterial endotoxin can activate different coagulation cascades in horseshoe crab amebocyte lysate. Endotoxin specifically activates factor $B$ and $C$ while BDG activates factor G. By removal of factor C from Limulus amebocyte lysate, coagulation cascade is activated only by BDG (Figure 1). 


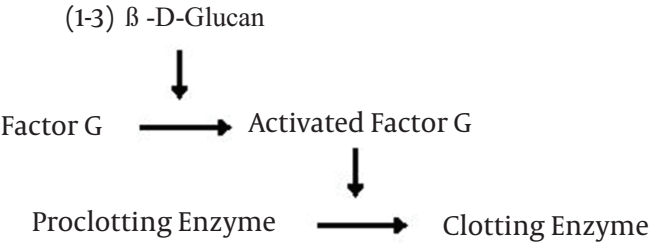

Figure 1. Glucan Effect on Limulus Amebocyte Lysate Coagulation Pathway

The activation rate of this coagulation cascade can be assessed and quantified by colorimetric or turbidimetric methods. Commercial assay systems to detect BDG apply different reagents derived from different species of horse shoe crabs, therefore these assays have different positive cutoff values (13). The method was established first in 1995 in Japan and then in 2004 in USA and has been recommended as one of the indirect mycological criteria to diagnose invasive fungal infection in the diagnostic guideline published by the European Organization for Research and Treatment of Cancer Invasive Fungal Infections Cooperative Group, and National Institute of Allergy and Infectious Diseases Mycoses Study Group [EORTC/ MSG] (14) in 2008.

It is well documented that presence of BDG in plasma or serum of at-risk patients is a valuable marker for invasive fungal infection (2). Nevertheless, routine application of this test has been problematic in many laboratories and the corresponding kits are approved in limited countries. The current study, in an attempt to evaluate usefulness of BDG assay, assessed the test by two different kits with different methods in two different laboratories based on a rat model of the experimental systemic candidiasis. The results of the experiment can provide useful preliminary data to improve and establish the BDG approaches in Iran and other developing countries.

\section{Objectives}

The present study aimed to evaluate $\beta$-D-Glucan assay in diagnosis and monitoring the systemic candidiasis in a rat model.

\section{Materials and Methods}

\subsection{Animals}

This study was performed according to the ethical principles for animal research (National Ethical Framework for Animal Research in Iran). Thirty three 8-12 week-old male rats (Sprague-Dawley), weighing $220 \pm 35$ g, were used for this study. Immunosuppression and neutropenia were induced in 27 out of 33 rats by intravenous administration of $200 \mathrm{mg} / \mathrm{kg}$ body weight of cyclophosphamide (Baxter Oncology GmbH Frankfurt, Germany) four days before inoculation and additional doses of $50 \mathrm{mg} /$ $\mathrm{kg}$ on the day of inoculation $(15,16)$. Six out of $27 \mathrm{immuno-}$ suppressed and six intact rats were used as the negative controls without any inoculations.

\subsection{Preparation of Yeast Cells for Injection}

A Candida albicans strain isolated from a patient with candidemia was used to prepare microbial suspension. The yeast was sub-cultured on Sabouraud dextrose agar (SDA, Merck KGaA, Darmstadt, Germany) plate for 48 hours at $37^{\circ} \mathrm{C}$ and harvested colonies were suspended in $10 \mathrm{~mL}$ sterile normal saline solution $(\mathrm{NaCl} 0.9 \%)$ and washed twice. Viable yeast cells in suspension were counted with a hemocytometer and the number of yeast cells adjusted to $1 \times 10^{6}$ per milliliter. To confirm the cell count, tenfold serial dilutions of suspension from $10^{6}$ to $10^{0}$ were prepared and 100 microliter of each dilution was spread onto SDA, and colonies were counted after 48 hours at $37^{\circ} \mathrm{C}$

\subsection{Induction of the Experimental Infection in the Rat Model}

A one hundred microliters aliquot $\left(1 \times 10^{5}\right.$ cells $)$ of $C$. albicans suspension was injected into the tail vein of 21 rats. During a period of seven days after inoculation, every twenty four hours three rats were anesthetized with chloroform and their cardiac puncture blood was collected in both EDTA free and EDTA coated tubes. Clotted blood was centrifuged for 5 minutes at 1300 rpm and the supernatant (serum) was separated and kept in $-20^{\circ} \mathrm{C}$ until use. The course of infection was evaluated by weighing the rats every day and assessing their weight loss as well as the yeast burden in their internal organs after sacrificing the animals.

\subsection{Pathological Studies}

Brain, heart, kidneys, spleen and liver of each rat were removed and about $0.5 \mathrm{~g}$ of each organ was homogenized in $2 \mathrm{~mL}$ of sterile normal saline. One hundred microliters of blood and the homogenate of rat organs were cultured on SDA plates for 48 hours at $37^{\circ} \mathrm{C}$ and then colonies were counted. Each homogenate was also examined microscopically.

Glucan assay: Beta-D-Glucan levels in all sera samples were assessed with two kits (Fungitell and Wako): Fungitell assay (Associates of Cape Cod Inc., Falmouth, Mass, Associates of Cape Cod Inc., Falmouth, Mass, USA) determines levels of BDG in serum or plasma. The detection reagent is a biological cascade based upon modified Limulus amebocyte lysate (LAL), an extract of the blood cells of the North American horseshoe crab. The Fungitell reagent is modified to eliminate factor $\mathrm{C}$ and, thus bypassing the activated factor $\mathrm{B}$, only reacts to $\mathrm{BDG}$, through the factor G-mediated side of the pathway. This renders the reagent highly specific for BDG and does not react to other polysaccharides, including beta-glucans with different glycosidic linkages. 
Khodadadi H et al.

Table 1. Approximate Number of Candida CFU per 100 Microliter of Tissue Homogenates and Blood Cultured on SDA Plates

\begin{tabular}{lcccccccccccccccccccccc}
\hline Rat Number & $\mathbf{1}$ & $\mathbf{2}$ & $\mathbf{3}$ & $\mathbf{4}$ & $\mathbf{5}$ & $\mathbf{6}$ & $\mathbf{7}$ & $\mathbf{8}$ & $\mathbf{9}$ & $\mathbf{1 0}$ & $\mathbf{1 1}^{\mathrm{a}}$ & $\mathbf{1 2}$ & $\mathbf{1 3}$ & $\mathbf{1 4}$ & $\mathbf{1 5}^{\mathrm{a}}$ & $\mathbf{1 6}^{\mathrm{a}}$ & $\mathbf{1 7}$ & $\mathbf{1 8}$ & $\mathbf{1 9}$ & $\mathbf{2 0}$ & $\mathbf{2 1}$ \\
\hline Kidney & $10^{2}$ & $<10$ & $10^{5}$ & $10^{1}$ & 105 & $<10$ & $10^{4}$ & $10^{5}$ & $10^{5}$ & $10^{3}$ & - & $10^{3}$ & $10^{5}$ & $10^{3}$ & - & - & $10^{4}$ & $10^{5}$ & $10^{4}$ & $10^{5}$ & $10^{5}$ \\
Spleen & $10^{1}$ & $<10$ & $10^{4}$ & $10^{2}$ & $10^{3}$ & $<10$ & $<10$ & $10^{5}$ & $10^{5}$ & $10^{4}$ & - & $10^{3}$ & $10^{4}$ & $10^{2}$ & - & - & $10^{2}$ & $10^{4}$ & $10^{2}$ & $10^{4}$ & $10^{4}$ \\
Liver & $<10$ & 0 & $10^{1}$ & $<10$ & $10^{1}$ & $<10$ & $10^{1}$ & $10^{2}$ & $10^{3}$ & $10^{1}$ & - & $<10$ & $10^{2}$ & $<10$ & - & - & $10^{1}$ & $10^{2}$ & $10^{1}$ & $10^{2}$ & $10^{2}$ \\
Heart & $<10$ & 0 & $10^{3}$ & $<10$ & $10^{2}$ & 0 & $<10$ & $10^{3}$ & $10^{4}$ & $10^{1}$ & - & $<10$ & $10^{3}$ & $<10$ & - & - & $10^{2}$ & $10^{2}$ & $10^{3}$ & $10^{4}$ & $10^{1}$ \\
Brain & 0 & 0 & $10^{1}$ & $<10$ & 0 & 0 & $<10$ & $10^{2}$ & $10^{2}$ & $<10$ & - & $10^{2}$ & $10^{2}$ & $<10$ & - & - & 0 & $10^{1}$ & $10^{1}$ & $10^{2}$ & $10^{1}$ \\
Blood & $<10$ & 0 & 0 & 0 & 0 & 0 & $<10$ & 0 & 0 & 0 & - & 0 & 0 & 0 & - & - & 0 & $<10$ & 0 & $<10$ & $<10$ \\
\hline
\end{tabular}

a Rats number 11,15 and 16 died during the experiment.

The assay was fulfilled according to the manufacturer instruction. Briefly, $5 \mu \mathrm{L}$ of serum or plasma was added to duplicate wells of a 96-well microtiter plate and pretreated with $20 \mu \mathrm{L}$ of serum treatment reagent (a 1:1 mixture of $0.125 \mathrm{M} \mathrm{KOH}$ and $0.6 \mathrm{M} \mathrm{KCl}$ ) for 10 minutes at $37^{\circ} \mathrm{C}$. Then $25 \mu \mathrm{L}$ of each standard (6.25 to $100 \mathrm{pg} / \mathrm{mL}$ Glucan) was added to each standard well. One hundred microliter of Fungitell reagent (Limulus amebocyte lysates with pyrosol as substrate) was added to each sample and standard or blank well. The rate of change of absorbance per minute ( $\Delta \mathrm{OD} / \mathrm{min}$ ) at $405 \mathrm{~nm}$ (with $490 \mathrm{~nm}$ background subtraction) was monitored for 40 minutes at $37^{\circ} \mathrm{C}$ by using a THERMO max automated microplate reader (Molecular Devices) equipped with SOFT max PRO software, version 3.1.1. A kinetic standard curve was constructed and BDG concentrations of unknown samples were interpolated from standard curve.

The beta-glucan Wako test (Wako Pure Chemical Industries, Osaka, Japan) also determines levels of BDG in plasma. The mechanism of the assay is the same as Fungitell assay with the difference that this assay uses turbidimetric detection method instead of colorimetric method. The procedure for this assay was conducted according to the manufacturer instruction. Briefly, $100 \mu \mathrm{L}$ of plasma was added to BDG free tubes containing $900 \mu \mathrm{L}$ of pretreatment solution (0.2\% triton X-100, $0.002 \%$ polymyxin B). Tubes were incubated at $80^{\circ} \mathrm{C}$ for 10 minutes to neutralize any contaminating endotoxin. After cooling the tubes for 5 minutes, $100 \mu \mathrm{L}$ of the supernatant of pretreated samples was added and mixed with $100 \mu \mathrm{L}$ of LAL reagent. In this process, BDG activates the coagulation reaction cascade. Increased turbidity was determined with the kinetic turbidimetric method using Toxinometer MT 251 (Wako Pure Chemical Industries, Wako Pure Chemical Industries, Osaka, Japan). The coagulation time (Tc) was defined as the reaction time required for the transmittance to diminish the threshold values (98\% of the initial transmittance). Therefore the higher the level of BDG in the sample, the shorter the coagulation time, and log (BDG) is inversely proportionate with $\log (\mathrm{Tc})$. Manufacturer has recommended a cutoff value of $11 \mathrm{pg} / \mathrm{mL}$ for this assay.

In order to validate the precision and repeatability of Fungitell assay, intra-assay (within run assay), and inter- assay (between run assays) were evaluated. To assess inter-assay variabilities the tests of all 30 sera samples of infected and non- infected rats were repeated on three different days. Intra-assay variabilities of the Fungitell assay were assessed by retesting three different serum samples, one serum with lower cutoff range of manufacturer $(19.5 \mathrm{pg} / \mathrm{mL})$, one in approximate range of cutoff value $(71.88 \mathrm{pg} / \mathrm{mL})$, and one with a higher range than the cutoff value, $(646 \mathrm{pg} / \mathrm{mL})$ in quadruplicate in a single assay. Replicate of each sample was placed in different locations within the microtiter plate. The mean and coefficients of variation (CVs) for replicates of the three samples were calculated. To evaluate glucan assay, all 30 sera samples were analyzed in Medical Mycology Laboratory of Tehran University of Medical Sciences (TUMS) with Fungitell kit and were also sent to Teikyo University, Institute of Medical Mycology, Tokyo, Japan (TIMM) to determine BDG concentrations with Wako kit.

\section{Results}

Three rats died during the experiment and after intravenous inoculation with $1 \times 10^{5} \mathrm{CFU}$ of C. albicans. The remaining 30 rats were sacrificed after cardiac puncture according to the ethical principles for animal research.

\subsection{Organ Deposition of C. albicans Blastospors}

Distribution of yeast in internal organs of rats, according to the colony count of homogenates cultured on SDA, was summarized in Table 1 . The severity of infection increased in heart, brain, liver, spleen and kidneys respectively. Only five blood samples cultured on SDA plates showed colonies of yeast having low colony counts, however, all rats showed organ involvement with C. albicans.

\subsection{Evaluation of Fungitell and Wako Assay in Rat Serum}

Using the predefined cutoff value of $80 \mathrm{pg} / \mathrm{mL}$ for Fungitell and $11 \mathrm{pg} / \mathrm{mL}$ for Wako, 18 sera samples of the 19 rats with the systemic candidiasis that had high level of BDG with a mean BDG concentration of 738.41 (ranged 28 to $1288 \mathrm{pg} / \mathrm{mL}$ ) were assessed with Fungitell, and the mean concentration 632.7 (ranged 5 to $3103 \mathrm{pg} / \mathrm{mL}$ ) was assessed with Wako. 
Khodadadi H et al.

\begin{tabular}{lcccc}
\hline \multicolumn{4}{l}{ Table 2. Comparing the Efficiency of Fungitell and Wako Kits Based on Newly Calculated Cutoff Values a,b } \\
\hline - Sensitivity & Specificity & PPV & NPV \\
\hline Fungitell & 95 & 66.6 & 90.47 & 80 \\
Wako & 90 & 83.3 & 94.7 & 71.4 \\
\hline a Abbreviations: NPV; Negative Predictive Value; PPV: Positive Predictive Value & & \\
b Data are presented as \%. & & &
\end{tabular}

\subsection{Fungitell Assay Reproducibility}

The inter-assay coefficients of variances (CVs) for the three runs were $5.8 \%$ to $20.9 \%$, and $32.1 \%$, for the serum samples with BDG concentrations of $19.5 \mathrm{pg} / \mathrm{mL}, 71.88$, and 646, respectively. The mean inter-assay CV for all 30 samples was $19.6 \%$. The intra-assay CVs for the three tested samples were $3.3 \%$ and $5.8 \%$ for the samples with BDG concentrations of 19.5 and $71.88 \mathrm{pg} / \mathrm{mL}$, respectively. Sample with BDG values of $646 \mathrm{pg} / \mathrm{mL}$ had replicate CVs of $11.6 \%$.

\subsection{Determination of the BDG Cutoff Value for Fun- gitell Assay}

The serum samples collected from 12 non-infected rats (six immunocompromised rats without infection and six intact rats) were assessed by Fungitell, tested at TUMS, had a mean BDG concentration of 93.4 pg/mL (range 16.8-157 $\mathrm{pg} / \mathrm{mL}$ ) and out of these 12 samples, five serum samples had $>80 \mathrm{pg} / \mathrm{mL}(84,87,117,143,157 \mathrm{pg} / \mathrm{mL})$ BDG concentration. By repeating the assay two more times (inter-assay) similar results were obtained with a mean CV around 8.3\% for all of the control samples. Infected rats had high level of BDG with a mean BDG concentration of $738.41 \mathrm{pg} / \mathrm{mL}$. Thus $120 \mathrm{pg} / \mathrm{mL}$ was used as positive cutoff value to discriminate BDG positive samples from the negative ones. Using Wako assay non-infected rat sera had a mean BDG concentration of $4.2 \mathrm{pg} / \mathrm{mL}$ (rang 2.5-27.4). Non-infected rat sera samples did not have BDG concentration $>27.4$ $\mathrm{pg} / \mathrm{mL}$. Intra- and inter-assays were not done on the control sera by this kit. The mean BDG value for the infected rat sera was $632.70 \mathrm{pg} / \mathrm{mL}$ (rang 5-3103 pg/mL). Predefined cutoff value of $11 \mathrm{pg} / \mathrm{mL}$ was used.

Diagnostic efficiency: With the calculated positive cutoff value $>120 \mathrm{pg} / \mathrm{mL}$ for Fungitell assay the sensitivity, specificity, Positive Predictive Value (PPV), and Negative Predictive Value (NPV) of the Fungitell were 95\%, 66.6\%, $90.47 \%$ and $80 \%$ respectively (Table 2 ) and with the positive cutoff value $>11 \mathrm{pg} / \mathrm{mL}$ the sensitivity, specificity, PPV, and NPV of the Wako assay were 90\%, 83.3\%, 94.7\% and $71.4 \%$ respectively (Table 2 ).

\section{Discussion}

Measurement of BDG has been recommended in some guidelines from Japan, Europe and USA to diagnose IFIs. The commercial kits used in these countries have different measurement methods (colorimetric or turbidimet- ric) and cutoff values thus sensitivity, specificity, PPV and NPV reported for each kit and for each experiment have shown large differences; then judgment on positive and negative results could be a laborious work leading to several clinical problems (17). Since a lot of factors could interfere with the results, it is very important that each laboratory sets up the test and calculates its own cutoff values and other diagnostic efficiency criteria.

The current study evaluated applicability of two BDG assays (Fungitell and Wako) to diagnose and monitor an IFI (systemic candidiasis) in an experimental model in order to use them as adjunctive tools for IFIs diagnosis in patients in Iran. The current experiment used sera of immunocompromised rats inoculated with C. albicans as proven IFI case group and sera of intact rats as the control group. Development of IFI (systemic candidiasis) in rats was approved by cultivation of homogenized organ suspensions on SDA and their microscopic examination. According to the results stated in Table 1, deposition of C. albicans blastospors in Spargue-Dawelly organs was different from those of blastospors in $\mathrm{BalB} / \mathrm{C}$ and $\mathrm{CBA}$ mice reported previously (18), and burden of infection increased in heart, brain, liver, spleen and kidneys respectively.

To evaluate the efficiency of these two assays the current study considered $120 \mathrm{pg} / \mathrm{mL}$ as cutoff value for Fungitell assay in the experiments. This cutoff value is higher than those recommended by manufacturer $(80 \mathrm{pg} / \mathrm{mL})$. Pezos et al. used $120 \mathrm{pg} / \mathrm{mL}$ as positive cutoff value in their experiments, too (12). Using this cutoff value, the sensitivity, specificity, PPV and NPV of Fungitell assays were 95\%, $66.6 \%, 90.47 \%$ and $80 \%$, respectively. According to the predefined cutoff value of $11 \mathrm{pg} / \mathrm{mL}$ by Wako assay, the sensitivity, specificity, PPV and NPV were 90\%, 83.3\%, 94.7\% and $71.4 \%$, respectively. These results are comparable to the ones published by Yoshida et al. (17), and look reliable. Of course Karageorgopoulos et al. in his Meta-analysis study reported a total sensitivity of $77 \%$ and specificity of $85 \%$ that shows a lower sensitivity than specificity for BDG assay (19).

False positive BDG results observed in the current experiment decreased significantly when the cutoff value was corrected to $120 \mathrm{pg} / \mathrm{mL}$. Some interfering conditions such as the dusty environment in Iran could change negative results to false positive. But this assumption should be approved with more studies. Coefficients of variances for inter and intra assays (Mean CVs 19.6\% and 6.9\% respec- 
tively) showed a poor reproducibility performance for Fungitell kit especially on measurement of BDG concentration higher than the cutoff value. In summary, the current study suggests that measuring BDG levels in serum of a patient suspected to IFIs is a helpful and adjunctive tool to diagnose and monitor the disease if the below point is considered as other researchers have noted (19, 20).

A frequency of two tests per week should be performed on each patient. Results of the BDG assay should be interpreted by a companion clinical, radiological and other laboratory criteria for the diagnosis of IFIs. Each laboratory should calculate its own cutoff value and perform quality control programs to qualify the applied kit to reduce false positive and negative results. Proper use of the test depends on the experienced technologist who performs the assay, and maximum reduction of the specimens contamination with gauze, environmental dusts and organic wastes in the lab.

\section{Acknowledgements}

Authors wish to appreciate Tehran University of Medical Sciences and Teikyo University Institute of Medical Mycology to have provided the opportunity to conduct the current study.

\section{Authors' Contribution}

Here we confirm that all authors participated in the research design and contributed during research.

\section{Financial Disclosure}

None declared.

\section{Funding/Support}

This study was not funded.

\section{References}

1. Clark TA, Hajjeh RA. Recent trends in the epidemiology of invasive mycoses. Curr Opin Infect Dis. 2002;15(6):569-74.

2. Pickering JW, Sant HW, Bowles CA, Roberts WL, Woods GL. Evaluation of a (1->3)-beta-D-glucan assay for diagnosis of invasive fungal infections. J Clin Microbiol. 2005;43(12):5957-62.

3. Lin SJ, Schranz J, Teutsch SM. Aspergillosis case-fatality rate: systematic review of the literature. Clin Infect Dis. 2001;32(3):358-66.

4. Odabasi Z, Mattiuzzi G, Estey E, Kantarjian H, Saeki F, Ridge RJ, et al. Beta-D-glucan as a diagnostic adjunct for invasive fungal infections: validation, cutoff development, and performance in patients with acute myelogenous leukemia and myelodysplastic syndrome. Clin Infect Dis. 2004;39(2):199-205.

5. Ellepola AN, Morrison CJ. Laboratory diagnosis of invasive candi- diasis. J Microbiol. 2005;43 Spec No:65-84.

6. Singh N, Paterson DL. Aspergillus infections in transplant recipients. Clin Microbiol Rev. 2005;18(1):44-69.

7. Berenguer J, Buck M, Witebsky F, Stock F, Pizzo PA, Walsh TJ. Lysiscentrifugation blood cultures in the detection of tissue-proven invasive candidiasis. Disseminated versus single-organ infection. Diagn Microbiol Infect Dis. 1993;17(2):103-9.

8. Mitsutake K, Miyazaki T, Tashiro T, Yamamoto Y, Kakeya H, Otsubo $\mathrm{T}$, et al. Enolase antigen, mannan antigen, Cand-Tec antigen, and beta-glucan in patients with candidemia. J Clin Microbiol. 1996;34(8):1918-21.

9. Jones BL, McLintock LA. Impact of diagnostic markers on early antifungal therapy. Curr Opin Infect Dis. 2003;16(6):521-6.

10. Stevens DA. Diagnosis of fungal infections: current status. J Antimicrob Chemother. 2002;49 Suppl 1:11-9.

11. Marty FM, Koo S. Role of (1->3)-beta-D-glucan in the diagnosis of invasive aspergillosis. Med Mycol. 2009;47 Suppl 1:S233-40.

12. Pazos C, Ponton J, Del Palacio A. Contribution of (1->3)-beta-Dglucan chromogenic assay to diagnosis and therapeutic monitoring of invasive aspergillosis in neutropenic adult patients: a comparison with serial screening for circulating galactomannan. J Clin Microbiol. 2005;43(1):299-305.

13. Miyazaki T, Kohno S, Mitsutake K, Maesaki S, Tanaka K, Ishikawa $\mathrm{N}$, et al. Plasma (1-->3)-beta-D-glucan and fungal antigenemia in patients with candidemia, aspergillosis, and cryptococcosis. $J$ Clin Microbiol. 1995;33(12):3115-8.

14. De Pauw B, Walsh TJ, Donnelly JP, Stevens DA, Edwards JE, Calandra $\mathrm{T}$, et al. Revised definitions of invasive fungal disease from the European Organization for Research and Treatment of Cancer/Invasive Fungal Infections Cooperative Group and the National Institute of Allergy and Infectious Diseases Mycoses Study Group (EORTC/MSG) Consensus Group. Clin Infect Dis. 2008;46(12):1813-21.

15. van Deventer AJ, Goessens WH, van Belkum A, van Vliet HJ, van Etten EW, Verbrugh HA. Improved detection of Candida albicans by PCR in blood of neutropenic mice with systemic candidiasis. $J$ Clin Microbiol. 1995;33(3):625-8.

16. Polak A. Experimental models in antifungal chemotherapy. Mycoses. 1998;41(1-2):1-30.

17. Yoshida K, Shoji H, Takuma T, Niki Y. Clinical viability of Fungitell, a new (1-->3)-beta-D: -glucan measurement kit, for diagnosis of invasive fungal infection, and comparison with other kits available in Japan. J Infect Chemother. 2011;17(4):473-7.

18. Ashman RB, Papadimitriou JM. Murine candidiasis. Pathogenesis and host responses in genetically distinct inbred mice. Immunol Cell Biol. 1987;65 ( Pt 2):163-71.

19. Karageorgopoulos DE, Vouloumanou EK, Ntziora F, Michalopoulos A, Rafailidis PI, Falagas ME. beta-D-glucan assay for the diagnosis of invasive fungal infections: a meta-analysis. Clin Infect Dis. 2011;52(6):750-70.

20. Lamoth F, Cruciani M, Mengoli C, Castagnola E, Lortholary O, Richardson $\mathrm{M}$, et al. beta-Glucan antigenemia assay for the diagnosis of invasive fungal infections in patients with hematological malignancies: a systematic review and meta-analysis of cohort studies from the Third European Conference on Infections in Leukemia (ECIL-3). Clin Infect Dis. 2012;54(5):633-43. 